\title{
Gastric Anatomic Type Is Associated with Obesity and Gender
}

\author{
Yu Jen Wang ${ }^{a}$ Kun-Long Hung ${ }^{\text {b, c }}$ Jui-Neng Yang ${ }^{d}$ Tien-Cheng Wang ${ }^{e}$ \\ Chih-Hui Chin ${ }^{\mathrm{e}}$

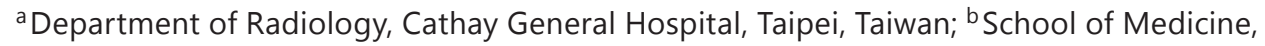 \\ Fu-Jen Catholic University, New Taipei City, Taiwan; ' Neurological Department, Cathay

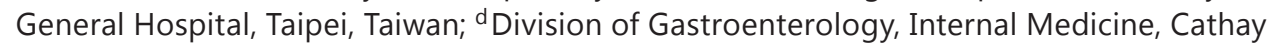 \\ General Hospital, Taipei, Taiwan; ${ }^{\mathrm{e}}$ Department of Internal Medicine, Internal Medicine, \\ Cathay General Hospital, Taipei, Taiwan
}

\section{Key Words}

Obesity $\cdot$ Overweight $\cdot$ Body mass index $\cdot$ Stomach

\begin{abstract}
Objective: To enhance our understanding of the associations among gastric anatomy, obesity, and gender. Methods: 777 randomly selected participants received health checkups, including a series of radiographs of the upper gastrointestinal tract (UGI); the findings were linked with each corresponding subject's gender and BMI. We measured the length, angle, and different portions of the stomach with the subjects in the standing position using radiographs to classify all individuals into anatomic types 1 through 6 based on gastric morphology. The gastric morphology was identified based on the initial UGI examination: 166 followup UGI radiographs at $12 \pm 1.5$ months to evaluate whether the stability of gastric anatomy persisted over time. Results: There was a significant difference in anatomic types between females and males $(p<0.001)$. The proportions of men with certain types (e.g., barium initially pools in a retroflexed fundus) were significantly higher than those of women; these participants were more likely to be overweight/obese $(p<0.001)$ compared with participants with other anatomic types. Additionally, the proportion of women with gastroptosis was significantly higher than that of men; participants with this type were less likely to be overweight/ obese ( $p$ 0.001). Conclusion: Gastric anatomic types were associated with obesity and gender.


Wang et al.: Gastric Anatomic Type Is Associated with Obesity and Gender

\section{Introduction}

Overweight and obesity, which belong to the most serious public health challenges of the 21 st century, create a major risk for serious diet-related chronic diseases [1]. The aim of this study was to investigate whether differences in gastric anatomy are associated with obesity and gender in adults. Kusano et al. [2] demonstrated that gastric morphology is related to BMI and gender. In subjects with cascade stomach (CS), swallowed barium initially pools in the retroflexed gastric fundus and fills it; afterwards the barium 'cascades' into the body of the stomach. BMI was significantly higher in both men and women with CS than in the respective controls [2]. Radiography of the upper gastrointestinal tract (UGI) has in the past constituted part of the typical health checkup in Japan [2] and at our hospital. After endoscopy became popular, the use of radiographic examination declined [2].

The stomach consists of five main topographic regions: the cardia, fundus, body, antrum, and pylorus. The proximal stomach is an area of special interest for the transient relaxation of the lower esophageal sphincter and its likely modulation [3], which creates a reservoir [4] for the meal and enables the gastric volume to increase. This process, which is called gastric accommodation, is a vagally controlled reflex and acts on the proximal stomach $[5,6]$. Differences in the properties and functions between the proximal and distal stomach have been recognized in human studies; the distal stomach is less compliant to low-level distension than the proximal stomach [7]. In the proximal stomach (i.e., the fundus), contraction waves spread slowly $(<1 \mathrm{~cm} / \mathrm{s})$ and are weak, allowing some mixing of ingested food and gastric secretions and, more importantly, facilitating food storage. The waves are stronger and faster (traveling $3-4 \mathrm{~cm} / \mathrm{s}$ ) in the antrum [8]. A study in mice demonstrated that the mechanosensitivity of the gastric vagal afferent nerves is significantly reduced after receiving a high-fat diet (HFD) and that the gastric tension receptors are reduced; consequently, the responses to distension are also decreased [9]. Another study found that the disruption of gastric vagal afferent function by HFD-induced obesity is only partially reversible by dietary change [10], which suggests that if a person has a disturbance in the gastric vagal afferent function, he/she would need to eat more food before feeling the same degree of fullness than a healthy individual [10]. Thus, the increase in the chamber/radius of the proximal stomach may be caused by a disruption in the vagal afferent function, allowing a person to eat more; the results may change based on gastric anatomy and increases in BMI. However, managing ingested nutrients in the gastrointestinal tract is a complex process that is closely regulated by both humoral and neural mechanisms [11]. Keeping in mind a literature review of recent articles, the present study was designed to evaluate the associations between gastric anatomy, BMI, and gender.

\section{Material and Methods}

\section{Inclusion Criteria}

A total of 777 participants between the ages of 19 and 75 years ( $n=447$ men and 330 women) received health checkups, which consisted of chest radiographs, complete blood examinations, a series of doublecontrast UGI radiographs, and simple physical examinations; additionally, gender and BMI of all subjects were recorded at our hospital from June 2008 through December 2010. The 777 participants were randomly selected from among 3,000-3,300 subjects/year, who were evaluated from June 2008 through December 2010. These participants received health checkups voluntarily or in compliance with their employers and government regulations; they were not referred to our facility because of upper gastrointestinal symptoms. The patients were referred to the appropriate specialists within a week for additional management if abnormalities were identified. Several participants continued to return voluntarily for additional health checkups. We studied 166 participants who returned for follow-up radiographic UGI series. Informed consent was obtained from all of the study participants. The study protocol was approved by the ethics committee and research board of our hospital (Cathay General Hospital) and was in accordance with the Declaration of Helsinki. 

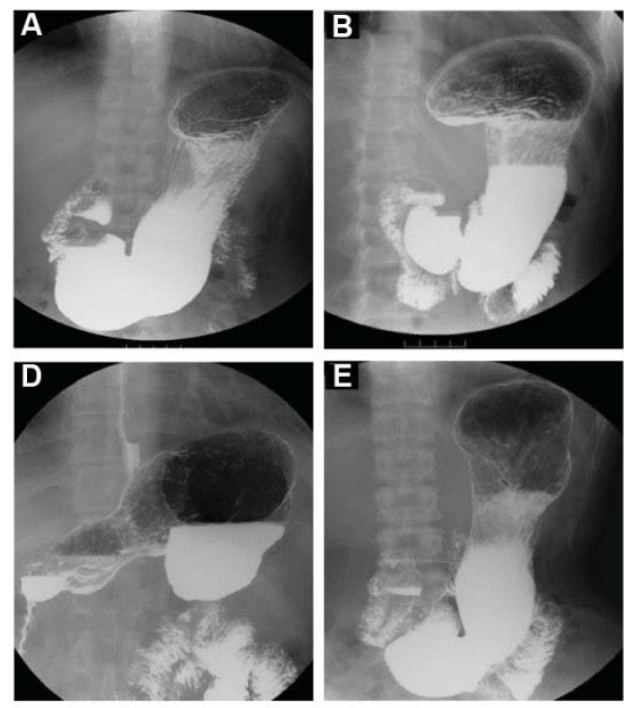
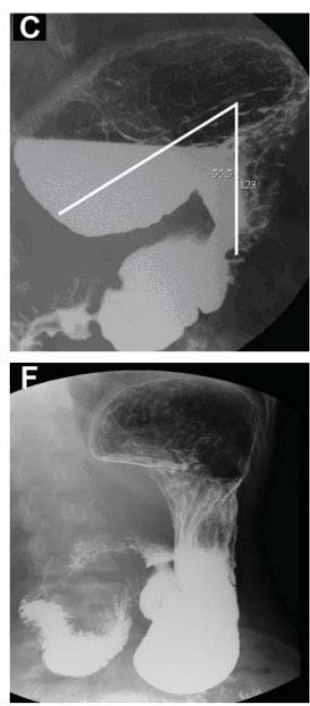

Fig. 1. Definition of the gastric anatomic subtypes. A (A1): Either the platform over the medial fundus is less than $3.8 \mathrm{~cm}$ in length (or is not discernible) or the angle of the medial fundus portion axis and body axis of the stomach is more than $100^{\circ}$. B (A2): The platform of the medial fundus (horizontal base) is more than 3.8 $\mathrm{cm}$ in length, and the angle of the medial fundus and body axis of the stomach must be between $80^{\circ}$ and $100^{\circ}$; this type may allow barium meal stasis on the platform. C (A3): The platform of the medial fundus has an upward facing concave shape; the horizontal line of the concave up platform is more than $3.8 \mathrm{~cm}$ in length, and the angle of medial fundus axis and body axis of the stomach must be less than $80^{\circ}$. There is easy retention of barium in the fundus. D (A4): The fundus is upside down. For A1, A2, A3, and A4, the gastric base must be either above the level of the iliac crest or must not exceed $2 \mathrm{~cm}$ in length below iliac crest. $\mathbf{E}$ (A5): The platform over the medial fundus is less than $3.8 \mathrm{~cm}$ in length or not discernible, and the angle of the medial fundus portion axis and body axis of the stomach must be more than $100^{\circ}$. The lower base of the lower gastric portion (antrum) that drops into the pelvic cavity must be situated below the level of the iliac crest and must always exceed $2 \mathrm{~cm}$ in length. F (A6): The lower base of the lower gastric portion drops into the pelvic cavity, must be situated below the level of the iliac crest, and must always exceed $2 \mathrm{~cm}$ in length; otherwise, the criteria for the fundus and body are the same compared to type 2 .

\section{Exclusion Criteria}

Participants were excluded from the study if they had any precipitating factors that might be associated with restriction in the gastrointestinal tract, such as prior nasogastric intubation, ingestion of medications (such as aspirin or non-steroid anti-inflammatory agents) within the recent 3 months, and inadequate distention of the stomach in the imaging study (e.g. due to a patient's hiccup). Participants with a history of gastrointestinal surgery were also excluded from the study because the gastric morphology of these patients could be caused by a pre-existing condition. There were no pregnant participants in our series.

\section{Observations and Measurements during UGI Radiography}

Our main concern in this study was to examine the relationships among the maximal radius of the fundus, gender, and BMI. We applied the same study procedure to all participants. All patients fasted for at least $8 \mathrm{~h}$ before the double-contrast UGI. Each participant was administered approximately $180 \mathrm{ml}$ of barium meal together with $4 \mathrm{~g}$ of effervescent granules (Top; Taejoon Pharmaceuticals, Kyungkido, South Korea) with a small amount of water (approximately $12 \mathrm{ml}$ ) before the examination to achieve adequate gastric distention and morphology. We performed several maneuvers during each UGI series, including filming the flow of the barium meal into the esophagus, through the stomach and into the duodenum from different positions. Buscopan ${ }^{\circledR}$ (scopolamine butylbromide) was not used in our study because Buscopan exerts a parasympathicolytic action [12]; if used, the peristalsis of the observed gastrointestinal tract would be unnatural. We evaluated the UGI findings based on the real-time fluoroscopic and workstation imaging analyses (IMPAX DS3000; Agfa, Mortsel, Belgium). 
Wang et al.: Gastric Anatomic Type Is Associated with Obesity and Gender

Fig. 2. A demonstration of A1. The platform over the medial fundus is not discernible; the gastric base is not below the level of the iliac crest and does not exceed $2 \mathrm{~cm}$ in length. The maximal radius of the proximal stomach (fundus) is 8.8 cm in length in this patient.

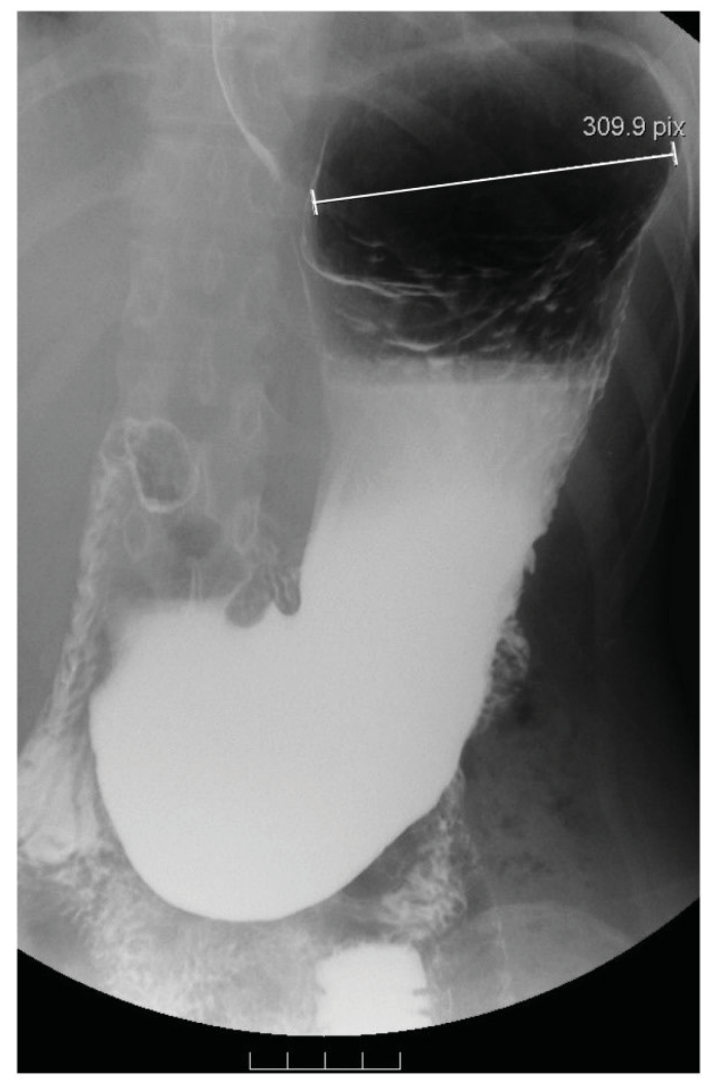

Measurement of the Maximal Radius of the Fundus and Angle between the Medial Fundus and Body of the Stomach (If Present)

Gastric imaging reveals the maximal radius of the gastric fundus and the angle between the fundus axis and body axis, thus allowing measurement of the length of the medial fundus platform (should this region be present in any participant). The subjects were in the standing position in the final stage of the UGI study, either on anterior-posterior projection or occasionally the right anterior oblique view (RAO), especially for CS, to obtain the maximal radius of the fundus; similarly as described by Kusano et al. [2]; 0-50 ${ }^{\circ}$ in our series. The angle (if present) between the medial fundus axis and the body axis was assessed by drawing lines along these structures on the radiographs. The subject's body position was sometimes slowly adjusted under fluoroscopy to obtain an ideal view (particularly for anatomical types 2 and 3) (fig. 1-4).

The WHO criteria [13] were applied: normal weight $\geq 18.5-24.9 \mathrm{~kg} / \mathrm{m}^{2}$, overweight $\geq 25-29.9 \mathrm{~kg} / \mathrm{m}^{2}$, and obesity $\geq 30-39.9 \mathrm{~kg} / \mathrm{m}^{2}$. Our study is based on the WHO criteria.

\section{Statistical Analysis}

The associations of anatomic types with gender and BMI were evaluated using Pearson's chi-square or Fisher's exact test. Additionally, the chi-square test for trends was used to investigate the ordinal variables. The maximal radius of the stomach is presented as the median (inter-quartile range) and was compared across the anatomic types using a Kruskal-Wallis test and Mann-Whitney U post hoc test because the maximal radius of the stomach was not normally distributed. All statistical assessments were two-sided, and $\mathrm{p}<0.05$ was considered significant. An adjusted significance level of $0.0167(0.05 / 3)$ was also considered for the post hoc pairwise comparisons. Statistical analyses were performed using SPSS (Statistical Package for the Social Sciences) 15.0 statistics software (SPSS Inc., Chicago, IL, USA). 
Fig. 3. A demonstration of A3. Stasis of a barium meal over the platform of the medial fundus in air-fluid level appearance; the horizontal line of the platform (concave up shape) is approximately $4.8 \mathrm{~cm}$ in length of the medial fundus; the angle of the medial fundus axis and the body axis of the stomach is $53.4^{\circ}$. The maximal radius of the proximal stomach (fundus) is $11 \mathrm{~cm}$ in length.
Wang et al.: Gastric Anatomic Type Is Associated with Obesity and Gender
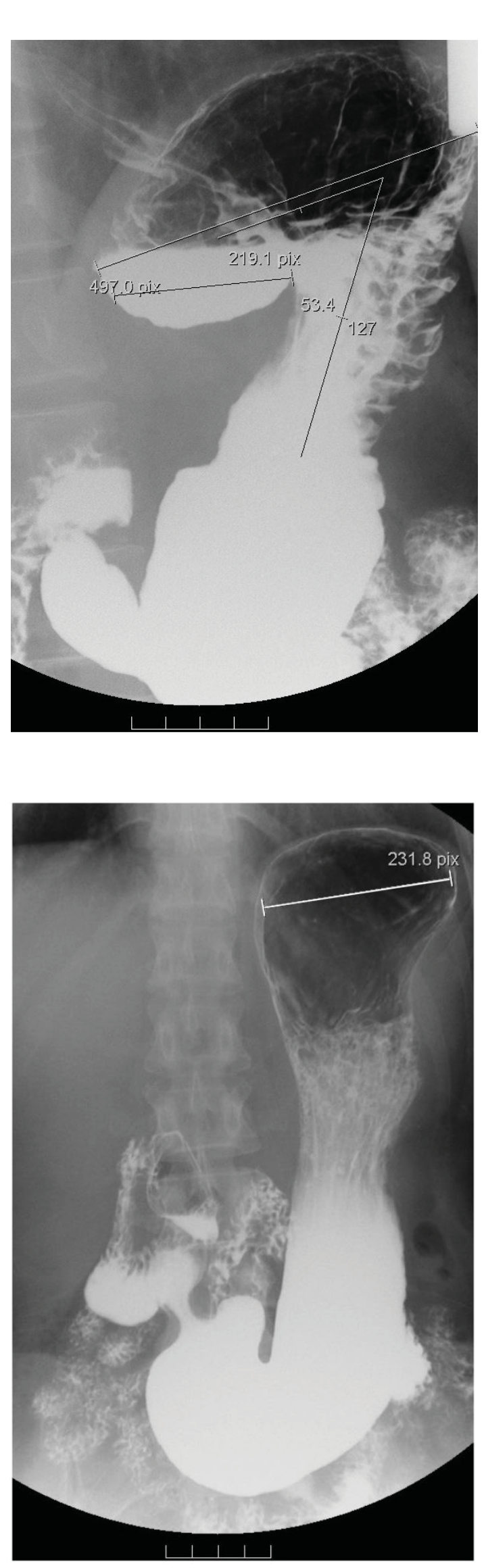

Fig. 4. A demonstration of A5. The platform over the medial fundus is not discernible; the lower base of the lower gastric portion (antrum) drops into the pelvic cavity, must be situated below the level of the iliac crest, and must always exceed $2 \mathrm{~cm}$ in length. The maximal radius of the proximal stomach (fundus) is $7.8 \mathrm{~cm}$ in length in this patient. 
Wang et al.: Gastric Anatomic Type Is Associated with Obesity and Gender

Table 1. Relationships among anatomic type, gender, and BMI $(\mathrm{n}=777)^{\mathrm{a}}$

\begin{tabular}{|c|c|c|c|c|c|c|c|}
\hline \multirow[t]{2}{*}{ Anatomic type } & \multicolumn{2}{|c|}{ Gender, n (\%) } & \multirow[t]{2}{*}{$\mathrm{p}$ value } & \multicolumn{3}{|l|}{ BMI, n (\%) } & \multirow{2}{*}{ p value } \\
\hline & women & men & & $\begin{array}{l}\text { normal } \\
\left(\mathrm{BMI}<25 \mathrm{~kg} / \mathrm{m}^{2}\right)\end{array}$ & $\begin{array}{l}\text { overweight } \\
\left(\text { BMI } 25-30 \mathrm{~kg} / \mathrm{m}^{2}\right)\end{array}$ & $\begin{array}{l}\text { obesity } \\
\left(\mathrm{BMI}>30 \mathrm{~kg} / \mathrm{m}^{2}\right)\end{array}$ & \\
\hline Type $1(\mathrm{n}=342)$ & $104(31.5)$ & $238(53.2)$ & $<0.001^{*}$ & $214(41.1)$ & $115(50.9)^{\dagger}$ & $13(43.3)$ & 0.046 \\
\hline Type $2(\mathrm{n}=143)$ & $26(7.9)$ & $117(26.2)$ & $<0.001^{*}$ & $75(14.4)$ & $59(26.1)^{\dagger}$ & $9(30)^{\dagger}$ & $<0.001^{*}$ \\
\hline Type $3(n=54)$ & $7(2.1)$ & $47(10.5)$ & $<0.001^{*}$ & $20(3.8)$ & $27(11.9)^{\dagger}$ & $7(23.3)^{\dagger}$ & $<0.001^{*}$ \\
\hline Type $4(n=7)$ & $1(0.3)$ & $6(1.3)$ & 0.249 & $0(0)$ & $7(3.1)^{\dagger}$ & $0(0)$ & 0.001 \\
\hline Type $5(n=228)$ & $190(57.6)$ & $38(8.5)$ & $<0.001^{*}$ & $210(40.3)$ & $17(7.5)^{\dagger}$ & $1(3.5)^{\dagger}$ & $<0.001^{*}$ \\
\hline Type $6(n=3)$ & $3(0.9)$ & $0(0)$ & 0.076 & $3(0.6)$ & $0(0)$ & $0(0)$ & 0.607 \\
\hline
\end{tabular}

aThe frequency of individuals with each anatomic type is shown as $\mathrm{n}(\%)$. The differences were determined using either Pearson's chi-square or Fisher's exact test (i.e., if the sample size was $<5$ ).

${ }^{*} \mathrm{p}<0.05$ indicates a significant difference between the given characteristics.

${ }^{\dagger} \mathrm{p}<0.0167$ indicates a significant difference in the normal BMI values compared with the dispersion of anatomic types in BMI values.

Table 2. Relationship between anatomic types and the maximal radius of the proximal stomach (fundus) $(\mathrm{n}=777)^{\mathrm{a}}$

\begin{tabular}{llll}
\hline Anatomic type & Maximal radius & p value & Post hoc $^{\#}$ \\
\hline Type $1(n=342)$ & $8.7(7.5-9.7)$ & $<0.001^{*}$ & type 3 , type $2>$ type 1 \\
Type $2(\mathrm{n}=143)$ & $9(8-10)$ & & \\
Type $3(\mathrm{n}=54)$ & $9.65(8.35-11.43)$ & \\
Type $4(\mathrm{n}=7)$ & $10(8.8-10)$ & \\
Type $5(\mathrm{n}=228)$ & $8(7.2-8.9)$ &
\end{tabular}

The data are presented as the median values (inter-quartiles, Q1Q3) for anatomic types and were compared using the Kruskal-Wallis test and Mann-Whitney U post hoc test because the data were not normally distributed.

${ }^{*} p<0.05$ indicates a significant difference among the anatomic types.

${ }^{\#}$ The post hoc test was significant at the $\mathrm{p}=0.008$ level.

\section{Results}

Six Anatomical Stomach Types (fig. 1A-E); All 777 Patients Could Be Divided into the Six Types Based on the Standing Position

We developed a new method for distinguishing 6 different stomach types. We found that all of 777 patients could be included into the 6 types. Other specific types, such as gastric volvulus, were not observed in our UGI series during the checkup.

Anatomic type 1 was the most common overall characteristic. Anatomic type 5 was the most common characteristic in women. Significantly more men than women were identified as having anatomic type 1,2 or 3 (each $\mathrm{p}<0.001$ ).

Participants who were overweight and obese were more likely to have anatomic types 2 and 3 than those with BMI $<25 \mathrm{~kg} / \mathrm{m}$.

Most of the participants with anatomic type 5 had normal BMI, in contrast to other types. Anatomic types 4 and 6 were rare (less than 2\%) (table 1).

The differences in the anatomic types were correlated with the differences in the maximal radius of the fundus. The radius of the fundus was greater in anatomic types 3 and 2 than in type 1 (table 2). 


\section{How Stable Were These Six Types over Time?}

We were concerned that the stomach appearance in the radiograph could be affected by factors related to diet, BMI, etc. A total of 166 of the 777 subjects had UGI series follow-up at $12 \pm 1.5$ months to determine whether the gastric anatomic type was similar at two different time points. Anatomic changes were identified in only 7 (less than 5\%) of the 166 participants between the two performed studies. The gastric anatomy appeared to be stable over time (12 \pm 1.5 months). Indeed, in 3 of 7 individuals the anatomical changes were accompanied by body weight changes of more than $5 \mathrm{~kg}$ between the two time points. The factors responsible for anatomical changes over time may require in-depth investigation and long-term followup.

\section{Discussion}

Abdominal or intra-abdominal adiposity, which represents visceral fat within the abdominal cavity, may be more frequently observed in men than in women [14]. A greater proportion of men have android (i.e., upper body) fat distribution than premenopausal women; however, a significantly lower proportion of premenopausal women have android fat compared with postmenopausal women [15]. Evaluation of intra-abdominal fat could be important for assessing gastric anatomy [2], and hormonal factors should be investigated in the future to determine whether or not these factors are responsible for the significant difference in anatomic types between females and males.

Our study is not the first to assess the type and shape of the stomach. The aim of this study was to stimulate new ideas with respect to the differences among BMI, gender, and gastric anatomic types. An earlier study had established classification exclusively for patients without any organic radiologically detectable (non-operated) stomach lesions [16]. However, their study did not include BMI and obesity. UGI radiography is a non-invasive procedure because the examination allows physicians to directly observe the patient's gastric anatomy in real time. However, radiation exposure is a major concern. Anatomic type 3 is a typical type of CS [2]. Anatomic types 2 and 3 may be consistent with the CS, excluding a subset of the subjects with anatomic type 2 who exhibit an angle of the medial fundus and body axis of the stomach exceeding $90^{\circ}$.

In the present study, we provide one likely explanation for the changes in gastric morphology regarding increased BMI, which might be related to the increase of the maximal radius of gastric fundus (such as due to disruption of gastric vagal afferent function), and proved another potential effect (i.e., differences in the properties and functions between the proximal and distal stomach) [7-10]. We found that if an individual's BMI increases above the cut-off values indicating overweight or obesity, the proximal stomach may extend to the lesser sac (such as anatomic type 3) (fig. 5) because other parts of the stomach are surrounded by solid organs (i.e., the spleen, liver, pancreas, and diaphragm) and have difficulties extending at these sites. In our opinion, the key point for determining the length of the maximal radius of fundus and angulations between the fundus and the body of types 2 and 3 could be mainly based on the relative positions between the gastric body and the protruding part of the fundus toward the lesser sac. However, this topic requires additional investigation.

\section{Limitations}

First, these gastric anatomic types were identified based on our study. Our sample contained a limited number of racial and ethnic minorities. Additionally, radiation exposure is a major concern. However, the UGI series in the past has been part of health checkups in Japan [2] and at our hospital. Additional research aiming to validate these associations could 
Fig. 5. The territory of the proximal gastric fundus may extend into the surrounding mesentery and could eventually expand to the lesser sac, such as in anatomic type 3, particularly for participants with BMI above the cutoff values for overweight or obesity. The lesser sac ( + ) is lower in resistance to the extending portion of the gastric fundus, which may provide the space for platform formation over the medial fundus and for an increase of radius in length.

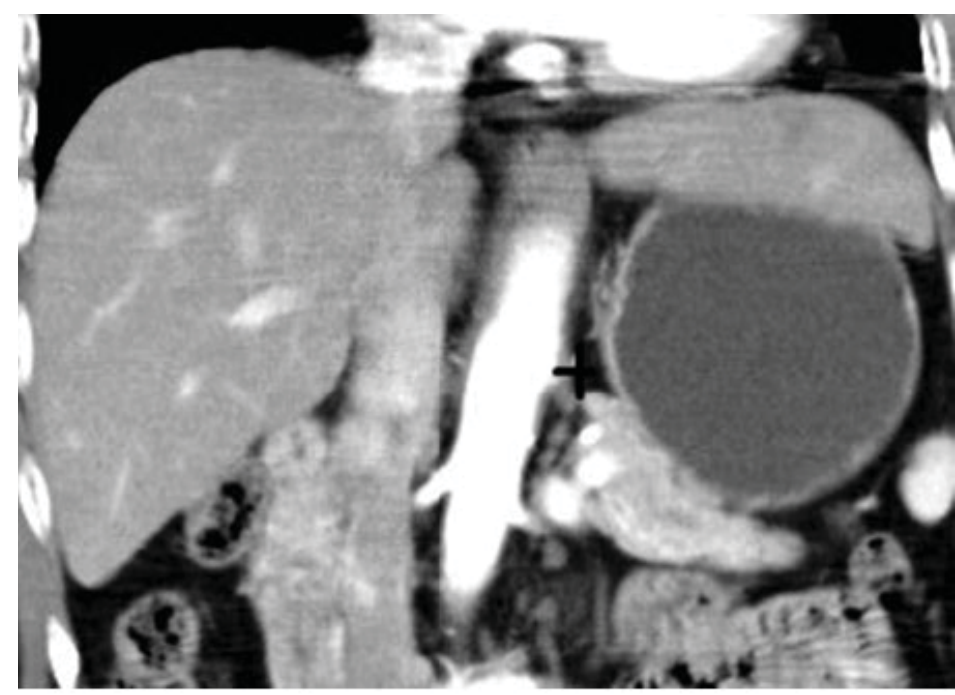

be performed using prior data (from recent decades). UGI radiography is presently not an advisable measure for screening because of radiation exposure. The use of radiographic examination has declined rapidly, so only 166 of 777 subjects had UGI series follow-up at 12 \pm 1.5 months. Looking for alternative methods may be necessary if additional research is required. Second, our study demonstrated a close relationship between BMI and gastric anatomy; however, we did not evaluate the diet of our participants. Hence, we could not conclude whether or not vagal injury played a role in the differences in gastric anatomy among different individuals based on the current study.

Managing ingested nutrients within the gastrointestinal tract is a complex process that includes the gastric responses to food intake as well as sensory and motor responses to gastric nutrients. This process is closely regulated by both humoral and neural mechanisms [11]. Our study only demonstrated the significant relationships between BMI, the maximal radius of the gastric fundus, gastric anatomic subtypes, and gender.

In conclusion, this study suggests that an increase in the radius of the gastric fundus over the proximal stomach may produce a change in the gastric anatomy, particularly in males; additionally, hormonal factors may need to be evaluated in women. The message we want to communicate to clinicians and researchers is that certain types of gastric anatomy are associated with obesity (types 2 and 3) and gender (types 2 and 3 for male; type 5 for female). The maximal radius in the fundus was greater in anatomic types 3 and 2 than in type 1.

\section{Acknowledgments}

The authors are grateful to C.C. Hwang (chief technician, Radiological Department, Cathay General Hospital) for his help in preparing the radiographs.

Funding support: None.

\section{Disclosure Statement}

The authors declare that they have no potential conflicts of interest to disclose. 
Wang et al.: Gastric Anatomic Type Is Associated with Obesity and Gender

\section{References}

1 Coll JL, Bibiloni Mdel M, Salas R, Pons A, Tur JA: Prevalence and related risk factors of overweight and obesity among the adult population in the Balearic Islands, a Mediterranean region. Obes Facts 2015;8:220-233.

2 Kusano M, Hosaka H, Moki H, Shimoyama Y, Kawamura O, Kuribayashi S, Mori M, Akuzawa M: Cascade stomach is associated with upper gastrointestinal symptoms: a population-based study. Neurogastroenterol Motil 2012;24:451-455.

3 Penagini R, Carmagnola S, Cantù P, Allocca M, Bianchi PA: Mechanoreceptors of the proximal stomach: role in triggering transient lower esophageal sphincter relaxation. Gastroenterology 2004;126:49-56.

4 Camilleri M, Vazquez-Roque M: Gastric dysmotility at the organ level in gastroparesis; in Parkman HP, McCallum RW (eds): Gastroparesis. New York, Humana Press, 2012, pp 37-46.

5 Vanden Berghe P, Janssen P, Kindt S, Vos R, Tack J: Contribution of different triggers to the gastric accommodation reflex in humans. Am J Physiol Gastrointest Liver Physiol 2009;297:G902-G906.

6 Troncon LE, Thompson DG, Ahluwalia NK, Barlow J, Heggie L: Relations between upper abdominal symptoms and gastric distension abnormalities in dysmotility like functional dyspepsia and after vagotomy. Gut 1995; $37: 17-22$.

7 Lee KJ, Vos R, Janssens J, Tack J: Differences in the sensorimotor response to distension between the proximal and distal stomach in humans. Gut 2004;53:938-943.

8 Johns Hopkins Medicine: Gastroparesis: Introduction. 2015. www.hopkinsmedicine.org/gastroenterology hepatology/_pdfs/esophagus_stomach/gastroparesis.pdf(last accessed June 7, 2016).

9 Kentish S, Li H, Philp LK, O’Donnell TA, Isaacs NJ, Young RL, Wittert GA, Blackshaw LA, Page AJ: Diet-induced adaptation of vagal afferent function. J Physiol 2012;590:209-221.

10 Kentish SJ, O'Donnell TA, Frisby CL, Li H, Wittert GA, Page AJ. Altered gastric vagal mechanosensitivity in dietinduced obesity persists on return to normal chow and is accompanied by increased food intake. Int J Obes (Lond) 2014;38:636-642.

11 Farré R, Tack J: Food and symptom generation in functional gastrointestinal disorders: physiological aspects. Am J Gastroenterol 2013;108:698-706.

12 de Brouwer EJ, Arbouw ME, van der Zwet WC, van Herwaarden MA, Ledeboer M, Jansman FG, ter Borg F: Hyoscine N-butylbromide does not improve polyp detection during colonoscopy: a double-blind, randomized, placebo-controlled, clinical trial. Gastrointest Endosc 2012;75:835-840.

13 Mbada CE, Adedoyin RA, Ayanniyi O: Socioeconomic status and obesity among semi-urban Nigerians. Obes Facts 2009;2:356-361.

14 Lagergren J: Influence of obesity on the risk of esophageal disorders. Nat Rev Gastroenterol Hepatol 2011;8: 340-347.

15 Ley CJ, Lees B, Stevenson JC: Sex- and menopause-associated changes in body-fat distribution. Am J Clin Nutr 1992;55:950-954.

16 Burdan F, Rozylo-Kalinowska I, Szumilo J, Zinkiewicz K, Dworzanski W, Krupski W, Dabrowski A: Anatomical classification of the shape and topography of the stomach. Surg Radiol Anat 2012;34:171-178. 